\title{
Valuing FSA research and development
}

\author{
Area of research interest: Capabilities \\ Project status: Completed \\ Conducted by: Risk solutions \\ DOI: https://doi.org/10.46756/sci.fsa.wyo220
}

\section{Background}

We fund research and development across a broad spectrum of areas aimed at improving public health and consumer confidence and developing more flexible approaches to regulation that deliver effective assurance.

The intended benefits from our funded research can be hard to measure and attribute, such as new food safety standards which may lead to positive social change.

Previous attempts to assess the benefits delivered by our research have met with limited success, and our own review of the literature concluded that none of the published valuation methodologies met our need. This project was commissioned to develop a new bespoke valuation methodology that could be used to inform the prioritisation of future research and development investment.

\section{Approach}

The work was carried out between October 2018 and January 2020 by Risk Solutions, with Live Economics Ltd and CECAN Ltd. An initial draft version of the RDVA was developed based on the findings of a literature review, workshops and interviews. This was then tested by project officers, who applied it to a sample of our projects, some in the early stages of planning and some that were currently 'live'. As well as helping to test and validate the research and development valuation approach, this resulted in a library of project case studies, which can be referenced by future research and development project officers.

\section{Results}

This work has developed a methodology for valuing our research and development projects, and other projects with benefits that are uncertain or difficult to describe. The process of completing the research and development valuation approach is designed to prompt deliberative thinking about the pathways to benefit and the design of the project.

It consists of the following elements:

- determination that the methodology applies

- categorisation of the project based on the fit with our strategic objectives and the research topic area

- provision of a brief narrative description, using a theory of change type structure, of how outputs from the research are intended to lead to intermediate outcomes and beneficial impacts through one or more delivery pathways

- scoring of semi-quantitative valuation criteria for research quality, potential research utility (timeliness and usability of outputs), potential reach and significance of impacts, and risk 
and reward balance.

Funding decisions for projects should then be based on consideration of both the narrative (which has limits on the length allowed) and the scores, within the wider context of our needs. The report provides recommendations for further developing the research and development valuation approach, and implementation and governance of the process.

Research report

PDF

View Valuing FSA research and development final report as PDF(Open in a new window) (1.14 $\mathrm{MB})$

PDF

View Valuing FSA research and development final report appendices as PDF(Open in a new window) (1.3 MB) 